\title{
Tuberculosis multirresistente
}

\author{
Drug resistant tuberculosis
}

Luis Eduardo Ramírez Bejarano(1), Julia Edith Chamorro Ortega ${ }^{(2)}$

\section{Resumen}

La tuberculosis es una enfermedad que a ha generado 1,4 millones de muertes por año en el mundo y que en América Latina sigue siendo una endémica, pese a los avances en los métodos diagnósticos y en el tratamiento. Adicionalmente, se ha visto un incremento de los casos de tuberculosis multirresistente a los antibióticos de primera línea, hecho que constituye un problema clínico y epidemiológico que conlleva elevada morbimortalidad y requiere intervención prioritaria de salud pública. El tratamiento para la tuberculosis multirresistente se basa en antituberculosos de segunda línea los cuales tienen mayor costo, mayores eventos adversos, y necesidad de tratamientos prolongados. Se presenta el caso clínico de paciente de sexo femenino, de 25 años de edad, con diagnóstico de tuberculosis pulmonar, con resistencia a fármacos de primera línea y complicaciones respiratorias y sistémicas severas, antecedente familiar de madre con diagnóstico de tuberculosis pulmonar 4 años previos con tratamiento completo y criterios de curación.

Palabras clave: tuberculosis, multirresistente, isoniazida, rifampicina.

\begin{abstract}
Tuberculosis is a sickness that in the world has generated 1,4 millions of dead people by year, and today tuberculosis in Latin America is an endemic sikcness despite improvements in treatments and diagnosis methods, else many cases of tuberculosis multi-resistant to the antibiotics of first line are seen these cases show us a big problema of Public Health Service. The treatment of these multi-resistant bacteria need new drugs, drugs called of second line and these drugs are more expensive,have more adverse events and treatments are longer. We have a clinic case of tuberculosis in a woman 25 years old. This patient with resistant treatment to antibiotics of first, many breaths problems; her mother had tuberculosis illness too 4 years ago; her mother received full basic treatment and after.. criterium of healing.
\end{abstract}

Keywords: tuberculosis, multi-resistant, isoniazid, rifampicina

\section{Introducción}

La tuberculosis es una de las enfermedades infecciosas responsables de las más altas tasas de morbimortalidad en el mundo, al punto de convertirse en un problema de salud pública. En 2011, se reportaron 8,7 millones de nuevos casos de tuberculosis activa en todo el mundo (13\% de los cuales estaba asociado con el virus de la inmunodeficiencia humana-VIH-) y 1,4 millones de muertes, incluyendo 430 mil, entre pacientes infectados por el VIH. Por otro lado, se estiman 310.000 casos nuevos de tuberculosis multirresistente (1, 2) asociados a múltiples factores entre los que se destacan: VIH y su asociación con enfermedad activa, expansión de la población, mala identificación y detección de casos, transmisión activa en cárceles y otros lugares públicos, migración de personas de países de alta incidencia, entre otros (2-4).

La fármacorresistencia plantea una amenaza particular a los programas nacionales contra la tuberculosis, ya que las pruebas de sensibilidad de las drogas no estás disponibles.
${ }^{(1)}$ Médico General, Fundación Universitaria San Martín.

${ }^{(2)}$ Especialista en Medicina Interna, Universidad del Cauca, Neumología Universidad Javeriana. Fundación para el cuidado del corazón y el pulmón.

Correspondencia:

Recibido: . Aceptado: 
En Colombia existen informes de resistencia global del 14,1\% y de multirresistencia del 1,8\% para el año 1992, del 15,6\% entre el año 1999 2000 para resistencia global y del 1,5\% para multirresistencia (5).

En el Reino Unido, alrededor del 8\% de los pacientes con tuberculosis tienen Mycobacterium tuberculosis resistente a los medicamentos de primera línea, el $6,9 \%$ a la isoniazida y el $1,4 \%$ a la rifampicina (6).

La aparición de la tuberculosis extremadamente multirresistente en el sur de África, y la aparición de amplia resistencia a los medicamentos en Europa del Este, ha dado lugar a nuevos análisis de la prevalencia mundial (6).

El resurgimiento de la tuberculosis con características epidémicas y la aparición de cepas multirresistentes, hace imperativo el diseño de estrategias de diagnóstico y estudios de susceptibilidad a drogas que permitan obtener resultados inmediatos.

\section{Caso}

Paciente femenina, de 25 años, con antecedente patológico de asma en manejo con salmeterol fluticasona, espirometría con reporte de alteración ventilatoria restrictiva y obstructiva severa, sin mejoría con el broncodilatador; antecedente quirúrgico de liposucción cinco meses previos, y hospitalización por neumonía basal izquierda tres meses atrás.

Consultó a un Hospital de Nivel III de atención, por cuadro clínico de sintomatología respiratoria conformado por disnea progresiva, hemoptisis leve, diaforesis nocturna y pérdida de peso; se documentó neumonía basal izquierda (figura 1), al examen físico TA 102/73, FC 120, FR 19, alerta, ruidos respiratorios disminuidos en hemitórax izquierdo, estertores crepitantes de predominio izquierdo, resto del examen normal; paraclínicos: leucocitosis 19.349, neutrofilia $78,8 \%$, trombocitosis 731,000 , PCR positiva, prueba rápida VIH negativa; cultivo de secreción bronquial: Enterobacter cloacae. Se inició manejo antibiótico con meropenem, con persistencia de respuesta inflamatoria sistémica, por lo que se solicitó fibrobroncoscopia la cual reportó endobronquitis severa con estenosis del bronquio fuente izquierdo (figura 2) con estudios de lavado bronquial positivo para BAAR ${ }^{+++}$. Se inició manejo antituberculoso de primera línea tetraconjugado: rifampicina, isoniazida, pirazinamida, etambutol, sin mejoría clínica, con deterioro de función hepática. Fue valorada por Infectología, que suspendió el manejo de primera línea. Se documentó multirresistencia (isoniazida y rifampicina) por lo cual se inició manejo con claritromicina, moxifloxacina, ácido paraminosalicílico y ampicilina sulbactam. Presentó atelectasia masiva pulmonar izquierda por estenosis bronquial persistente, por lo cual se decidió realizar manejo quirúrgico con neumonectomía radical izquierda.

\section{Discusión}

El caso corresponde a una mujer en la segunda década de la vida, previamente sana, quien consultó por cuadro de sintomatología respiratoria, documentándose tuberculosis multirresistente. La resistencia de Mycobacterium tuberculosis se observó después de la introducción de la estreptomicina en 1944; algunos casos han sido notificados desde 1977, pero a partir de 1987 se empezaron a notificar brotes de esta patología en diversas partes de Estados Unidos,

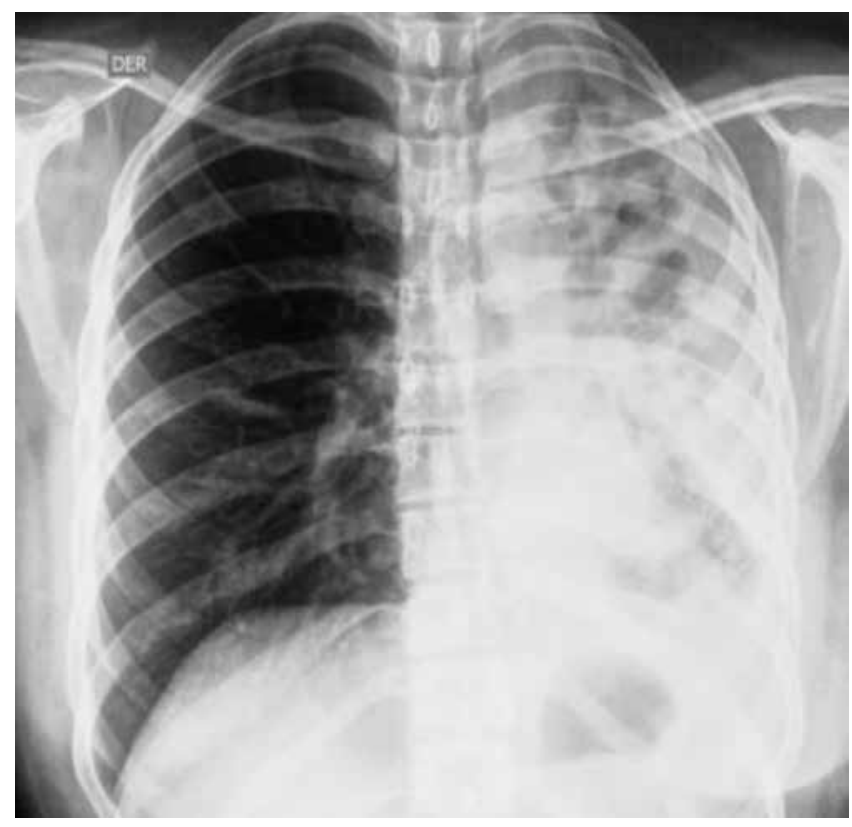

Figura 1. 


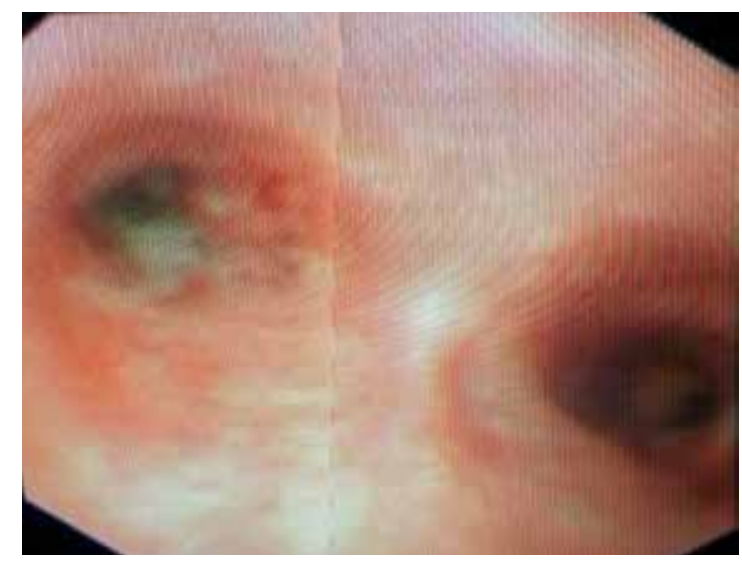

Figura 2.

principalmente en Nueva York. Las cepas resistentes se perpetúan por tratamiento inadecuado o suspensión del tratamiento por eventos de adversos $(7,8)$.

El término tuberculosis multirresistente se define cuando una cepa de Mycobacterium tuberculosis es resistente, como mínimo, a los efectos de la isoniazida y la rifampicina con o sin resistencia a cualquier otro medicamento.

La tuberculosis extremadamente multirresistente se define como la resistencia a alguna fluoroquinolona y a, por lo menos, uno de los tres fármacos endovenosos de segunda línea (capreomicina, kanamicina y amikacina), además de ser tuberculosis multirresistente $(1,4,8,9)$.

Se han establecido cuatro categorías diferentes de farmacorresistencia:

- Monorresistencia: resistencia a un fármaco antituberculoso.

- Polirresistencia: resistencia a más de un fármaco antituberculoso diferente de la isoniazida y la rifampicina.

- Multidrogorresistencia: resistencia a, por lo menos, isoniazida y rifampicina.

- Resistencia extendida a los fármacos: resistencia a alguna fluoroquinolona, y a, por lo menos uno de los tres fármacos inyectables de segunda línea (capreomicina, kanamicina y amikacina), además de multidrogorresistencia $(1,4,9)$.
La resistencia del Mycobacterium tuberculosis se debe predominantemente a alteraciones en la secuencia de nucleótidos en genes que codifican blancos de antibióticos, desarrollan resistencia a múltiples drogas por la acumulación de mutaciones individuales en varios genes, cada uno de los cuales es responsable de la resistencia a un antibiótico en particular (8). La caracterización de estas mutaciones ha conducido al desarrollo de nuevas estrategias de diagnóstico molecular que podrían acortar el periodo de reporte del patrón de resistencia a las drogas. La detección temprana de cepas resistentes a drogas de primera línea, contribuiría a un mejor manejo terapéutico del paciente con drogas de segunda línea y disminución del riesgo de propagación de cepas de tuberculosis multirresistente $(4,7,9,10)$.

La terapia de corta duración recomendada por la Organización de la Salud incluye los antibióticos de primera línea Categoría grupo I: isoniazida, rifampicina, pirazinamida, estreptomicina y etambutol. Lamentablemente, la estrategia de corta duración deja de ser la opción terapéutica para pacientes infectados con Mycobacterium tuberculosis multirresistentes $(8,10,11)$.

De otro lado, el tratamiento de cepas multirresistentes requiere medicamentos de segunda línea los cuales tienen más eventos adversos, mayores costos y tiempo de duración prolongado. Una limitación importante en la elección de la mejor estrategia de tratamiento para la tuberculosis multirresistente, es la escasez de ensayos controlados, y la mayoría de las recomendaciones se basan en revisiones retrospectivas, caso de serie u opinión de expertos.

Las recomendaciones del tratamiento para la tuberculosis multirresistente consisten en una fase intensiva de ocho meses, que incluye pirazinamida y un mínimo de cuatro fármacos de segunda línea: un antibiótico inyectable tuberculostático Categoría grupo II (kanamicina, amikacina, capreomicina, o viomicina), una fluoroquinolona de última generación Categoría grupo III (moxifloxacina, levofloxacina, ofloxacina), un tuberculostático bacteriostático Categoría grupo IV (etionamida, protionamida, cicloserina, teridizone y ácido para-aminosalicílico) y como última opción fármacos de eficacia dudosa 
para el tratamiento de la tuberculosis multirresistente Categoría grupo V (clofazimina, linezolid, amoxacilina/clavulanato, imipenem/cilastatina, claritromicina). El tratamiento debe ser continuo por un mínimo de veinte meses con cuatro fármacos eficaces, y la interrupción de la medicación inyectable al final del octavo mes de tratamiento si hay un buena respuesta clínica $(8,10,11)$.

Un tratamiento coadyuvante que ha demostrado aumento de la tasa de curación en relación con la utilización de tratamiento médico es la cirugía de tórax, la cual está indicada en pacientes con tuberculosis multirresistente o extremadamente resistente que tengan lesión localizada susceptible de resección, fracaso al tratamiento médico, alto riesgo de recaídas o complicaciones de la enfermedad (bronquiectasias, empiema, hemoptisis), en cuyos casos se han demostrado resultados favorables en el $89,5 \%$, si bien el momento óptimo de la cirugía adyuvante sigue siendo desconocido y no se ha abordado de manera adecuada en las pautas del tratamiento $(12,13)$.

Los patrones de resistencia en los diversos países y zonas, deben estudiarse y reportarse con rapidez, para permitirle al médico realizar un diseño correcto de regímenes terapéuticos eficaces, ya que la gran variabilidad de la resistencia, hace imposible una terapéutica uniforme en el mundo.

\section{Bibliografía}

1. World Health Organization Global tuberculosis control: WHO report 2012. Geneva: Disponible en: (http://apps.who.int/iris/bitstre am/10665/75938/1/9789241564502_eng.pdf)

2. Zumla A, Raviglione M, Hafner R, von Reyn F. Current concepts on tuberculosis. N Engl J Med. 2013; 368(8):745-55.

3. Frieden TR, Sterling TR, Munsiff SS, Watt CJ, Dye C. Tuberculosis. Lancet 2003;362:887-99.

4. Sharma SK, Mohan A. Tuberculosis: From an incurable scourge to a curable disease, ourney over a millennium. Indian J Med Res. 2013;137:455-93.

5. Miranda J, Ríos R, Clavijo A, Chacón C, Mattar S. Estudio preliminar de la susceptibilidad antimicrobiana y variabilidad genética de Mycobacterium tuberculosis en un área del Caribe colombiano. Colomb Med. 2006;37:275-86.

6. Maguire H, et al. Molecular epidemiology of tuberculosis in London 1995 7 showing low rate of active transmission. J Clin Pathol. 2003;56(2)121-6.

7. Edli BR, et al. An outbreak of multidrug-resistant tuberculosis among hospitalized patients with the acquired immunodeficiency syndrome. N Engl J Med. 1992;326:1514-21.

8. Field SK, et al. New treatment options for multidrug-resistant tuberculosis. Ther Adv Respir Dis. 2012;6(5):255-68.

9. Zumla A, et al. Drug resistant tuberculosis current dilemmas, unanswered questions, challenges, and priority needs. J Infect Dis. 2012;205(2):228-40.

10. Dooley KE, et al. Old drugs, new purpose: retooling existing drugs for optimized treatment of resistant tuberculosis. Clin Infect Dis. 2012;55(4):57281.

11. Lienhardt C, et al. New drugs for the treatment of tuberculosis: needs, challenges, promise, and prospects for the future. J Infect Dis. 2012;205(2):241-9.

12. Vashakidze S, et al. Favorable outcomes for multidrug and extensively drug resistant tuberculosis patients undergoing surgery. Ann Thorac Surg. 2013;95(6):1892-8.

13. Kempker RR, Vashakidze S, Solomonia N, Dzidzikashvili N, Blumberg HM. Surgical treatment of drug-resistant tuberculosis. Lancet Infect Dis. 2012;12(2):157-66. 